\title{
PORTRAIT PSYCHO-SOCIOLOGIQUE D'ETUDIANTS PRESENTANT DES TENDANCES SUICIDAIRES
}

\author{
MARTHE HURTEAU \\ Université de Montréal \\ et \\ YVAN BERGERON \\ Centre Action Suicide de Shawinigan
}

\section{RESUME}

Le présent article rapporte les résultats d'une étude effectuéc auprès des 550 étudiant(e)s du Cégep de Shawinigan inscrit(e)s en collégial 1. L'étude vise à établir la prévalence des comportements suicidaires et à décrire les facteurs circonstanciels en présence à savoir, les problèmes familiaux, le manque de support du réseau social, les éléments dépressifs, les événements stressants ayant un impact négatif, et les attitudes face à la vie et à la mort. L'information fut recueillie au moyen d'un questionnaire. Trois cent cinquante-cinq étudiant(e)s y ont répondu, fixant ainsi le taux de participation à $71 \%(355 / 550)$. Les sujets furent répartis en quatre groupes, soit les sujets ayant effectué une tentative de suicide $(n=28)$ ou une planification $(n=16)$; ceux ayant un score de 30 et plus au test portant sur les idéations suicidaires $(n=18)$ et, les sujets dits "normaux" ( $n=278)$.

Les sujets ayant déjả effectué une tentative de suicide rencontrent des problèmes familiaux importants. Ils n'ont pas de réel support du réseau social, présentent des tendances dépressives, et ont vécu des événements stressants qui ont eu un impact négatif sur leur vie. De plus, leur attitude en regard de la vie semble inadéquate à bien des égards. Les sujets ayant déjà planifié un suicide ne semblent pas présenter plus de problèmes que les sujets dits unormauxw, ce qui peut paraître assez étonnant à première vue. Une explication résiderait dans le fait que cette planification remonte loin dans le temps ou qu'elle a constitué un crì de détresse qui a trouvé une réponse. Quant aux sujets ayant un score de 30 et plus au test portant sur les idéations suicidaires, ils sont considérés à haut risque car ils présentent des problèmes assez similaires à ceux affichés par les personnes ayant déjà effectué une tentative de suicide. Finalement, les sujets dits «normaux» ne présentent pas de problème majeur, comme on serait en droit de s'attendre.

L'incidence du taux de suicides dans les pays industrialisés revêt des proportions de plus en plus inquiétantes. Au Canada seulement, après s'être maintenu stable jusqu'en 1960 , le taux a presque doublé au cours des 20 dernières années, passant de 7,6 à 14 par 100000 habitants. De plus, si jusqu'à tout récemment, la clientèle à plus haut risque suicidaire était constituée des personnes âgées, les Techerches de Grégoire et Phaneuf-Perron (1980), Charron (1981), ainsi que de Morissette (1984) confirment les données officielles (Santé et bien-être social, 1987) à l'effet qu'elle est maintenant remplacée par les jeunes de 15-24 ans. Les 
études de Lamontagne (1986) et de Tousignant, Bastien, Hamel, et Hanigan (1986) révèlent qu'un cégépien sur cinq a déjà planifié son suicide et qu'un sur 12 a déjà effectué une tentative. Le suicide est maintenant considéré comme la seconde cause de mortalité au Québec chez les jeunes, après les accidents d'automobiles qui pourraient s'avérer des suicides camouflés dans certains cas (Charron, 1981). Ces observations soulignent l'importance d'identifier les besoins spécifiques de cette population afin d'élaborer des interventions préventives.

Le présent article rapporte les résultats d'une étude effectuée auprès de la clientèle étudiante du Cégep de Shawinigan. Cette région se situe, en effet, au quatrième rang au Québec en ce qui concerne le taux de mortalité attribuable au suicide (Morissette, 1984). L'étude vise à établir la prévalence du phénomène ainsí qu'à décrire ses caractéristiques.

\section{CADRE THEORIQUE}

Le suicide se définit comme l'acte par lequel une personne met volontairement fin à ses jours (Bonner \& Rich, 1987; Davidson \& Philippe, 1986; Faber, 1968; Pokorny, 1960; Quidu, 1970; White, 1963). Les auteurs ont identifié les difficultés familiales, l'isolement social, les éléments dépressifs, les événements stressants, et les idéations suicidaires comme les facteurs les plus couramment reliés au suicide chez les 17-29 ans (Davidson \& Choquet, 1972; Friedman, Gregory, Boeck, \& Difiore, 1987; Haim, 1969; Harlow, Newcomb, \& Bentler, 1986; Hertzman, 1985; Joan, 1986; King, 1986; Lamontagne, 1986; Tousignant et al., 1986). Le jeune suicidaire est issu en général d'un milieu familial particulièrement perturbé: absence de l'un ou des deux parents (décès, séparation, divorce, ou abandon); conflits familiaux; carence affective de la première enfance; pauvreté de la communication et des relations dans la famille; présence au sein de la famille de problèmes multiples tels l'alcoolisme, l'usage de drogues, les maladies psychiatriques, et les tendances suicidaires ou même les tentatives de suicide. L'absence de support du réseau social est aussi une difficulté fréquemment rencontrée qui amène les personnes à se sentir isolées et à se persuader qu'elles ne peuvent compter sur aucune forme de soutien extérieur. Le vécu dépressif, quant à lui, s'exprime par le biais de symptômes psychologiques, biologiques, et psychomoteurs particuliers (Morissette, 1984). Finalement, les événements stressants agissent plus comme des facteurs précipitants dans le processus suicidaire qu'une cause comme telle. Le deuil d'une personne significative, la séparation des parents, leurs attitudes de rejet, des problèmes financiers, des échecs, une perte amoureuse, la maladie, etc. sont autant de circonstances qui peuvent contribuer à alimenter le stress et pousser la personne dans un processus suicidaire. Ainsi, tout jeune présentant les caractéristiques ci-dessus mentionnées est susceptible, à un moment ou à un autre, d'effectuer une tentative de suicide si aucune intervention n'est entreprise pour lui venir en aide.

\section{METHODE}

La présente étude revêt les caractéristiques d'une étude épidémiologique de type analytique (Jenicek, 1975). Elle vise à déterminer la prévalence de comportements suicidaires ainsi qu'à déterminer les facteurs circonstanciels qui les ac- 
compagnent. Les problèmes familiaux, l'absence de support du réseau social, les tendances dépressives, les événements stressants, et les attitudes face à la vie et à la mort (idéations suicidaires) sont retenus comme facteurs circonstanciels dans le cadre de la présente étude.

\section{Mesures des facteurs}

Chacun des facteurs est mesuré au moyen d'un instrument validé et adapté au contexte québécois.

1. Les problèmes familiaux. Ils sont mesurés par le biais d'un questionnaire qui s'inspire de celui utilisé par Lamontagne (1986) et Tousignant et al. (1986). Le questionnaire élaboré permet de recueillir principalement de l'information sur la situation maritale des parents, la qualité des relations qui prévalent dans la famille, ainsi que la présence et la fréquence de problèmes dans la famille (alcoolisme, maladies psychiatriques, tendances suicidaires, et décès par accident ou maladie).

2. L'absence de support du réseau social. Le Social Support Questionnaire de Sarason, Levine, Basham, et Sarason (1983), traduit et adapté par De Man (1986), est retenu pour mesurer l'existence et la disponibilité d'un éventuel support social. L'instrument, composé de 27 items, permet de déterminer d'une part, le nombre de personnes-ressources qui assureraient un support aux répondants en cas d'éventuelles difficultés, et d'autre part, le degré de satisfaction des répondants vis-à-vis cet éventuel support. En guise d'exemple, les questions se formulent de la façon suivante: "Sur qui peux-tu réellement compter pour te faire des suggestions utiles qui te permettent d'éviter de faire des erreurs?» Les répondants peuvent identifier jusqu'à neuf personnes. Le degré de satisfaction en regard de cet éventuel support se mesure au moyen d'une échelle de type Likert $(1=$ très insatisfait[e], $6=$ très satisfait[e]).

3. Les éléments dépressifs. Le test d'Aneshensel, Clark, et Fredrichs (1983), qui s'inspire du Center for Epidemiologic Studies Depression Scale (CESD Scale) de Radloff (1977), mesure l'état dépressif passager (au cours de la dernière semaine) et chronique des répondants. Les 20 items du test, traduits et adaptés par Tousignant et al. (1986), déterminent le niveau des affects positifs et négatifs, de la motivation et de la relation déficitaire.

4. Les événements stressants. Le Life Experience Survey de Sarason, Johnson, et Siegel (1978), traduit et adapté par De Man (1986), est composé de 57 items qui évaluent l'impact de certains événements sur la vie des répondants. Sur les 57 items du test, 47 s'adressent à l'ensemble de la population et 10 sont spécifiquement adaptés aux étudiants. Les répondants doivent identifier les événements qu'ils ont vécus au cours des 12 derniers mois précédant la passation du questionnaire ainsi que l'impact que ces événements ont eu sur leur vie (de -3 , événement perçu comme extrêmement négatif à +3 , événement perçu comme extrêmement positif).

5. Les attitudes face à la vie et à la mort. Les tentatives de suicide et les suicides complétés sont habituellement précédés d'idéations suicidaires (Morissette, 1984). Une connaissance de la gravité, de l'intensité, et des caractéristiques de ces idéations permet d'estimer l'intention et le risque suicidaires. Le Scale for Suicide Ideation de Beck, Kavacks, et Wiessman (1979) a été traduit et adapté par 
De Man (1986). Cette échelle mesure, au moyen de 19 items, le désir actif et passif de commettre un suicide (rapport vie/mort), ainsi que la préparation du suicide. Deux questions ont été ajoutées pour les besoins de la présente étude: (a) «As-tu déjà fait une tentative de suicide?»; et (b) «Si oui, à quand remonte la dernière tentative?»

De Man (1986) a eu recours à des procédures rigoureuses dans la traduction du test de Sarason et al. (1983) ainsi que celui de Beck et al. (1979). ${ }^{1}$

\section{Les sujets}

Contrairement aux études de Tousignant et al. (1986), Lamontagne (1986), et Bouchard (1987) qui ont eu recours à des échantillons, la présente étude a été effectuée auprès de la population des étudiants inscrits en collégial 1 au Cégep de Shawinigan. Le niveau collégial 1 a été sélectionné parce qu'il constitue une période de transition entre le secondaire et le collégial qui est souvent marquée par de nombreux bouleversements chez les jeunes. Effectivement, un grand nombre d'abandons est enregistré au cours de cette année. Ainsi, sur les 675 étudiants qui étaient inscrits en collégial 1 en septembre, il n'en restait que 500 en novembre, au moment de la cueillette des données.

\section{Mode d'enquête}

Le questionnaire administré aux cégépiens regroupe les cinq questionnaires énumérés précédemment. Il a fait l'objet d'une pré-expérimentation auprès d'une quinzaine d'étudiants, qui ne participaient pas à la présente étude, afin de vérifier la clarté des items et des directives ainsi que la pertinence des 50 minutes allouées à sa passation. Le questionnaire a été administré dans le cadre du cours de français, obligatoire pour tous les étudiants en collégial 1. L'anonymat des réponses était garanti et la participation s'est effectuée sur une base volontaire. Aucun refus n'a cependant été noté.

\section{PRESENTATION DES RESULTATS}

\section{Description générale de la clientèle}

Le taux de participation enregistré est de $71 \%(355 / 500)$. Les 145 sujets manquants étaient absents au cours de français au moment de la passation du questionnaire. Plus de $90 \%$ des étudiants interrogés sont originaires de Shawinigan et ils se répartissent en 202 adolescentes et 153 adolescents. Ce ratio respecte la distribution de la population étudiante du cégep. Les différentes concentrations sont aussi représentées de façon proportionnelle. L'orientation générale du Cégep de Shawinigan (secrétariat, éducation en garderie, sciences infirmières, etc.) explique, d'une part, le grand nombre de filles au cégep et, d'autre part, la faible proportion de clientèle extérieure. La majorité des étudiants vivent dans leur famille ( $68 \%$ ) et ils ont en moyenne 17 ans. Les analyses ont été effectuées sur 340 sujets, 15 d'entre eux ayant été éliminés à cause de valeurs manquantes.

1. Les problèmes familiaux. L'ensemble des répondants estiment les relations avec leurs parents comme très bonnes. II n'en demeure pas moins que les membres de certaines familles nucléaires sont aux prises avec des problèmes de 
divers ordres: $30 \%$ de décès dus à des accidents ou à la maladie, $18 \%$ d'alcoolisme, $10 \%$ de tentatives de suicide, et $6 \%$ d'antécédents psychiatriques. Ces problèmes existent dans des proportions trop importantes pour être ignorées.

2. Le support du réseau social. La moyenne globale obtenue au questionnaire portant sur le support du réseau social est de 2,64 personnes-ressources, soit moins de trois, et ce nombre varie peu d'un répondant à l'autre $(e ́-t=1,50)$. L'absence d'un support du réseau social constitue un problème généralisé à I'ensemble des répondants qui ne sont pas en mesure d'identifier au moins trois personnes sur lesquelles ils pourraient éventuellement compter en cas de crise. Cependant, ces mêmes répondants jugent l'éventuel support très satisfaisant, puisque la moyenne globale est de cinq sur une échelle de six degrés. Le père et la mère sont cités dans $85 \%$ des cas comme personnes-ressources. Cette observation peut étonner compte tenu de l'âge des répondants. En effet, on serait à même de s'attendre à ce que les pairs et les amis soient cités comme personnes pouvant constituer un éventuel réseau social.

3. Les éléments dépressifs. La moyenne globale au test d'Aneshensel et al. (1983) est de 37,5 sur une échelle de 80 points, indiquant ainsi de faibles tendances dépressives pour l'ensemble des sujets.

4. Les événements stressants. La moyenne globale au test portant sur l'impact des événements est de $-0,6$, indiquant ainsi que les répondants ont vécu durant la dernière année, plus d'événements ayant un impact négatif sur leur vie, que d'événements ayant un impact positif. Il faut dire que le résultat est tellement près de zéro qu'il constitue plutôt l'indication d'une absence d'impact, de neutralité. La dispersion des résultats (de $-34 \mathrm{a}+27$, avec un écart-type de 9,5) souligne une disparité dans le vécu des sujets.

5. Les attitudes face à la vie et à la mort. Les résultats portant sur les attitudes face à la vie et à la mort sont particulièrement intéressants car ils permettent de déterminer la prévalence du phénomène à l'étude. La moyenne globale est de 17 sur une échelle de 63 points, indiquant une faible tendance suicidaire au niveau de la population interrogée. L'écart-type d'une valeur de huit traduit cependant une variation importante dans les résultats et donc, la possibilité que certains individus soient à plus haut risque.

Finalement, tel qu'indiqué dans le tableau 1 , l'ensemble des variables sont en relation les unes avec les autres de façon significative $(p<, 05)$.

\section{Les sujets à risque}

Alors que les études portant sur le suicide jusqu'à ce jour se sont intéressées aux sujets ayant effectué une tentative ou une planification de leur suicide, la présente étude tente aussi d'identifier des sujets à risque auxquels font référence les auteurs lorsqu'ils parlent de personnes présentant des idéations et des ruminations suicidaires (Lamontagne, 1986; Morissette, 1984; Tousignant et al., 1986). Quatre groupes mutuellement exclusifs ont ainsi été formés.

Le groupe $A$ set composé de 28 répondants qui disent avoir effectué une tentative de suicide, dont huit au cours de la présente année.

Le groupe $B$ est composé de 16 répondants qui disent avoir déjà planifié un éventuel suicide. Cette catégorie sous-entend que la personne a déjà penser à un 
moyen concret pour mettre fin à ses jours et même possiblement un moment pour le faire.

Le groupe C est composé de 18 répondants qui n'ont effectué ni tentative de

\begin{tabular}{|c|c|c|c|c|}
\hline & Relatio & $\begin{array}{l}\text { ABLEAU } 1 \\
\text { entre les vari } \\
\mathbf{n}=340\end{array}$ & & \\
\hline & Support social & $\begin{array}{l}\text { Evénements } \\
\text { stressant }\end{array}$ & $\begin{array}{l}\text { Attitudes face } \\
\text { à la vie }\end{array}$ & $\begin{array}{r}\text { Tendances } \\
\text { dépressives }\end{array}$ \\
\hline Support social & 1,00 & & & \\
\hline $\begin{array}{l}\text { Evénements } \\
\text { stressants }\end{array}$ & $\begin{array}{l}, 17 \\
(, 001)\end{array}$ & 1,00 & & \\
\hline $\begin{array}{l}\text { Attitudes face } \\
\text { à la vie }\end{array}$ & $\begin{array}{l}-, 25 \\
(, 001)\end{array}$ & $\begin{array}{l}-, 31 \\
(, 001)\end{array}$ & 1,00 & \\
\hline $\begin{array}{l}\text { Tendances } \\
\text { dépressives }\end{array}$ & $\begin{array}{l}-, 20 \\
(.001)\end{array}$ & $\begin{array}{l}-, 46 \\
(, 001)\end{array}$ & $\begin{array}{l}, 43 \\
(, 001)\end{array}$ & 1,00 \\
\hline Moyenne globale & 2,72 &,- 52 & 16,87 & 37,42 \\
\hline Ecart-type & 1,49 & 9,55 & 7,64 & 11,12 \\
\hline
\end{tabular}

\section{TABLEAU 2}

Comparaison des résultats obtenus par les différents groupes concernant les relations avec les parents

\begin{tabular}{|c|c|c|c|c|c|c|}
\hline \multirow{4}{*}{$\begin{array}{l}\text { Groupes de } \\
\text { sujet } \\
(n=340)\end{array}$} & \multicolumn{6}{|c|}{ Relations avec les parents } \\
\hline & \multirow{2}{*}{\multicolumn{3}{|c|}{$\begin{array}{c}\text { Mère } \\
\text { Mauvaises }\end{array}$}} & \multicolumn{3}{|c|}{$\begin{array}{c}\text { Père } \\
\text { Manyalses }\end{array}$} \\
\hline & & & & & Mauvaises & \\
\hline & $\begin{array}{l}\text { Bonnes et } \\
\text { très bonnes }\end{array}$ & $\begin{array}{c}\text { et très } \\
\text { mauvaises }\end{array}$ & Inexistantes & $\begin{array}{l}\text { Bonnes et } \\
\text { très bonnes }\end{array}$ & $\begin{array}{c}\text { et très } \\
\text { mauvaises }\end{array}$ & Inexistantes \\
\hline Sujets ayant & 22 & 6 & - & 17 & 8 & 3 \\
\hline $\begin{array}{l}\text { effectué une } \\
\text { tentative } \\
(\mathrm{n}=28)\end{array}$ & $79 \%$ & $21 \%$ & & $61 \%$ & $29 \%$ & $10 \%$ \\
\hline Sujets ayant & 16 & - & - & 14 & 2 & - \\
\hline $\begin{array}{l}\text { effectué une } \\
\text { planification } \\
(\mathrm{n}=16)\end{array}$ & $100 \%$ & & & $88 \%$ & $12 \%$ & \\
\hline Sujets à & 14 & 4 & - & 10 & 7 & - \\
\hline $\begin{array}{l}\text { risque } \\
(\mathrm{n}=18)\end{array}$ & $78 \%$ & $28 \%$ & & $55 \%$ & $45 \%$ & \\
\hline Sujets & 259 & 5 & & 246 & 25 & 8 \\
\hline $\begin{array}{l}\text { normaux } \\
(\mathrm{n}=278)\end{array}$ & $93 \%$ & $4,5 \%$ & $3,5 \%$ & $88 \%$ & $9 \%$ & $3 \%$ \\
\hline Moyenne & & & & & & \\
\hline globale & & 4,39 & & & 4,17 & \\
\hline Ecart-type & &, 74 & & & ,90 & \\
\hline
\end{tabular}


suicide ni planification mais qui ont un score de 30 ou plus au test portant sur les idéations suicidaires. Ce groupe est considéré à risque dans le sens qu'ils pourraient présenter des idéations et ruminations suicidaires. ${ }^{2}$

Le groupe D est composé des 278 répondants dits "normaux».

\section{Description des groupes}

Les analyses qui suivent tentent d'établir la façon dont se comportent les divers groupes en regard de chacun des facteurs préalablement identifiés et de les comparer entre eux.

1. Les relations familiales. Les données du tableau 2 indiquent une bonne qualité des relations avec les parents et surtout avec la mère, pour la presque totalité des sujets des groupes B (planification) et D («normaux»). Cependant, si $80 \%$ des sujets du groupe A (tentative de suicide) estiment les relations avec la mère bonnes, ce pourcentage diminue à $60 \%$ en ce qui concerne les relations avec le père. Le même rapport peut être établi avec les sujets du groupe $\mathrm{C}$ (à risque) qui considèrent les relations avec leur mère bonnes dans $78 \%$ des cas, comparativement a $55 \%$ en ce qui concerne les relations avec leur père.

L'alcoolisme est présent dans $32 \%$ des familles, nucléaires en majorité, du groupe A (tentative de suicide), comparativement à environ $20 \%$ des sujets appartenant aux autres groupes. La moitié des sujets du groupe A (tentative de suicide) rapportent, d'une part, qu'un membre de leur entourage immédiat a effectué une tentative de suicide, comparativement à $22 \%$ des sujets du groupe $\mathrm{C}$ (à risque), $12 \%$ des sujets du groupe B (planification) et $4 \%$ du groupe D (cnormaux»); d'autre part, qu'ils ont vécu un décès, comparativement à $25 \%$-30 \% des sujets dans les autres groupes. Finalement, $21 \%$ des sujets du groupe A (tentative) identifient des antécédents psychiatriques dans leur famille comparativement d environ $5 \%$ des sujets dans les autres groupes. Toute proportion gardée, il appert évident que les problèmes familiaux sont plus nombreux et plus intenses chez les sujets du groupe A (tentative de suicide). Le taux élevé de suicides et de décés survenus dans leur famille immédiate semble très significatif et peut en partie expliquer le sens particulier que revêt ce geste pour eux.

2. Le support du réseau social. Les moyennes des divers groupes au questionnaire portant sur le support du réseau social varient entre 1,50 et trois personnesressources et les écarts-types sont faibles. Quant au taux de satisfaction vis-à-vis de l'éventuel support, les moyennes varient entre 4,3 et 5,4 sur une échelle de six. Aucune analyse de variance n'a été effectuée, étant donné le peu de dispersion des données. Les résultats obtenus peuvent s'expliquer en partie par le type de mesure utilisée. En effet, le questionnaire mesure le support du réseau social et non le réseau social comme tel. Ainsi, un jeune peut avoir beaucoup d'amis avec qui il sort, va au cinéma . . . mais peu de personnes sur qui compter pour recevoir un support. Ce phénomène peut aussi s'expliquer par le fait que le jeune qui doit demander de l'aide se perçoit "pas bon», ce qui l'amène à se dévaloriser par le fait même (Tousignant et al., 1986). Cette caractéristique se retrouve chez tous les jeunes et elle peut avoir des conséquences plus néfastes pour ceux qui sont aux prises avec de graves problemes. 
3. Les éléments dépressifs. Les moyennes obtenues au test d'Aneshensel et al (1983) varient passablement d'un groupe à l'autre. Ainsi, les sujets du groupe A (tentative de suicide) obtiennent une moyenne de 46,41 , ceux du groupe B (planification de suicide) obtiennent une moyenne de 36,17 , ceux du groupe $C_{(a)}$ risque) obtiennent une moyenne de 46,72 , et ceux du groupe D («normaux $)$ ) ob. tiennent une moyenne de 35,36 . L'analyse de variance s'avère significative $[F(3,335)=11,36, p<, 001]$, tel qu'indiqué dans le tableau $3 .{ }^{3}$ Les contrastes Scheffe a posteriori ont été effectués et les résultats obtenus indiquent certaines différences significatives $(p<, 01)$. Ainsi, les sujets ayant effectué une tentative de suicide (groupe A) et ceux considérés à risque (groupe C) présentent plus de tendances dépressives que les sujets des groupes B (planification du suicide) et D («normaux»).

4. Les événements stressants. Les résultats au test référant à l'impact des événements stressants indiquent que l'ensemble des groupes identifiés comme ayant des problèmes ont une moyenne négative. Ainsi, les sujets du groupe A (tentative de suicide) obtiennent une moyenne de $-5,73$, ceux du groupe B (planification) obtiennent une moyenne de,- 80 , ceux du groupe $\mathrm{C}$ (à risque) obtiennent une moyenne de $-5,89$, et seuls ceux du groupe D ( tiennent une moyenne positive de , 51 mais celle-ci est tellement faible qu'clle peut ètre interprétée comme une absence d'impact. L'analyse de variance s'avère significative $[F(3,329)=5,47, p<, 001]$, tel qu'indiqué dans le tableau 4 . Aucun contraste a posteriori ne s'avère significatif.

5. Les attitudes face à la vie et à la mort. Les résultats obtenus en ce qui concerne l'attitude face à la vie et à la mort varient énormément. Ainsi, les sujets du groupe A (tentative de suicide) obtiennent une moyenne de 30,17 , ceux du groupe B (planification) obtiennent une moyenne de 23,50 , ceux du groupe C (à risque) obtiennent une moyenne de 36,05 et ceux du groupe D («normaux $)$ ) obtiennent une moyenne de 14,44. L'écart des moyennes entre, d'une part, celles des groupes A (tentative de suicide) et B (planification de suicide) et, d'autre part, celle du groupe D («normaux») est remarquable. Il est évident que le groupe C obtient la moyenne la plus élevée puisque la variable étudiée constitue le critère de sélection des sujets de ce groupe (score au test sur les idéations égal ou plus élevé que 30 ). Cette situation pose par le fait même un problème au niveau de la comparaison entre les groupes et, dans cette perspective, il a été décidé d'effectuer une analyse de variance au niveau des deux groupes suivants: (a) les sujets ayant effectué une tentative ou une planification de suicide, qui ont donc posé un geste ou eu une pensée dans ce sens $(n=44)$ et, (b) l'ensemble des autres sujets $(n=296)$. Le premier groupe a obtenu une moyenne de 28,7 alors que la moyenne du second est 14,44 . La différence entre les moyennes s'avère significative $(F=130,76$, $p<, 001)$, tel qu'indiqué dans le tableau 5 .

\section{DISCUSSION}

Si l'ensemble des auteurs réfèrent dans leurs écrits à trois groupes pour décrire le phénomène du suicide, soit : (a) ceux qui ont fait une tentative; (b) ceux qui ont planifié leur suicide; (c) ceux qui ont des idéations suicidaires ou des ruminations suicidaires importantes, il n'en demeure pas moins cependant que leurs études jusqu'à ce jour ne portent que sur les deux premiers groupes. La 
présente étude a tenté d'identifier ce troisième groupe de sujets qui présentent des ruminations suicidaires et qui constituent par le fait même un risque élevé de passage à l'acte. Elle visait aussi à tracer un portrait des différents groupes et à les distinguer en regard de certains indicateurs de risque.

Le premier groupe est composé de sujets ayant déjà effectué une tentative de suicide (groupe A). Ces sujets rencontrent des problèmes familiaux importants. Pour plusieurs, les relations parentales, surtout avec le père, semblent détériorées. De plus, $50 \%$ d'entre eux ont eu connaissance du suicide ou du décès d'un des membres de leur entourage immédiat. Enfin, $25 \%$ ont des proches aux prises avec un problème de maladie mentale. Les écrits mentionnent que ces problèmes marquent souvent les jeunes (Chabrol, 1984; Davidson \& Choquet, 1972; Gaulthier, Fournier, \& Gorceix, 1961; Ladame, 1981; Morissette, 1984; Quidu, 1970; Tishler, Carl, McKenny, \& Morgan, 1981; Tousignant et al., 1986). A cela, s'ajoutent l'absence du support du réseau social et la présence d'éléments dépressifs et stressants. Il est cependant difficile de déterminer l'apport de chacun de ces facteurs dans leur première tentative. Comme celle-ci remonte à plusieurs mois dans la majorité des cas, la crise a de bonnes chances d'être résolue. Les besoins de certains d'entre eux auraient reçu l'attention nécessaire et les difficultés se sont par le fait même résorbées. Cependant, comme les idéations suicidaires

\section{TABI.EAU 3}

Analyse de variance concernant les tendances dépressives

\begin{tabular}{lcccc}
\hline Variable & $d l$. & $S c$ & $F$ & $p$ plus petit que \\
\hline $\begin{array}{l}\text { Tendances } \\
\text { dépressives }\end{array}$ & $\begin{array}{c}\text { variance }=3 \\
\text { expliquée } \\
\text { variance }=335 \\
\text { résiduclle }\end{array}$ & 1258,51 & 11,36 &, 001 \\
\hline
\end{tabular}

TABLEAU 4

Analyse de variance concernant les événements stressants

\begin{tabular}{lcccc}
\hline Variable & dl. & $S c$ & $F$ & $p$ plus petit que \\
\hline $\begin{array}{l}\text { Evénements } \\
\text { stressants }\end{array}$ & $\begin{array}{c}\text { variance }=3 \\
\text { expliquée } \\
\text { variance }=329 \\
\text { résiduelle }\end{array}$ & 482,25 & 5,48 &, 001 \\
\hline
\end{tabular}

TABLEAU 5

Analyse de variance concernant l'attitude face à la vie et à la mort

\begin{tabular}{lccccc}
\hline Variable & dl. & $S c$ & $F$ & $p$ plus petit que \\
\hline Attitude & $\begin{array}{c}\text { variance }=1 \\
\text { expliquée } \\
\text { variance }=344 \\
\text { résiduclle }\end{array}$ & 5543,43 & 130,76 &, 001 \\
\hline
\end{tabular}


semblent encore présentes dans certains cas, le suicide pourrait éventuellement être envisagé comme une solution pour résoudre rapidement et définitivement les difficultés (Morissette, 1984).

Le deuxième groupe est composé de sujets ayant planifié leur suicide (groupe B). Ces sujets ne semblent pas présenter plus de problèmes dans l'ensemble que le groupe D («normaux»), ce qui peut sembler assez étonnant à première vue, Une planification qui est maintenant assez éloignée dans le temps pourrait constituer une explication pour les résultats obtenus ou encore, ce geste a pu davantage constituer un cri d'alarme que la préparation concrète et claire de moyens pour s'enlever la vie. Dans cette éventualité, les répondants auraient reçu, au moment de la crise, le support nécessaire pour la transformer en une croissance person. nelle. Enfin, cela souligne aussi la nécessité, dans le cadre des prochaines études de préciser le terme de planification: Est-ce que cela signifie choisir la corde avec laquelle l'étudiant se pendra s'il coule son année académique ou est ce que cela signifie envisager prendre une dose fatale de barbituriques si jamais, un jour, il était aux prises avec une maladie incurable?

Les sujets ayant un score égal ou supérieur à 30 au test portant sur les idéa. tions suicidaires (groupe $\mathrm{C}$ ) ont été identifiés à risque sur la base de critères cliniques et descriptifs. Ceux-ci semblent effectivement présenter de nombreux problèmes. A celui des idéations suicidaires élevées, s'ajoutent des problèmes familiaux importants, de fortes tendances dépressives, ainsi que la présence de plusieurs événements stressants ayant un impact négatif sur leur vie. Le support d'un éventuel réseau social est mince et même s'il n'est pas vraiment présent dans aucun des groupes, il n'en demeure pas moins que les sujets du groupe demeurent plus vulnérables aux échecs dans ses relations (Davis, 1983; Farberow, 1985; Ladame, 1981; Tishler et al,, 1981; Withers \& Kaplan, 1987). L'accumulation d'événements stressants ayant un impact négatif (perte ou échec) conduit à ne pas investir dans de nouvelles expériences, ce qui provoque une perte d'estime de soi. Les éléments dépressifs présents peuvent déboucher sur un sentiment de désespoir, qui peut consolider les tendances suicidaires. Ces jeunes n'attirent pas l'attention sur eux et ils vivent leur désespoir à l'insu de tous (Carlson \& Cantwell, 1982; Chabrol, 1984; Corbeil, 1984; Ladame, 1981; Morissette, 1984; Teicher \& Jacobs, 1966). Ne suffirait-il pas d'une perte ou d'un échec parfois anodin pour les précipiter dans le processus suicidaire dont la mort est l'issue? On décrit souvent ces personnes, en rétrospective lorsqu'elles sont passées à l'acte, comme isolées, parlant peu et s'organisant sans déranger. Elles peuvent très bien réussir et être actives dans leur milieu. Parce qu'elles parlent très peu de leur vécu, le passage à l'acte est toujours surprenant et le premier geste est souvent le bon. Elles ne sont malheureusement pas considérées dans les études sur le suicide parce qu'elles n'ont jamais planifié ou fait une tentative de suicide. Cependant, certaines d'entre elles peuvent constituer des «bombes à retardement».

Finalement, les sujets dit «normaux» ne présentent pas de problème majeur comme on serait en droit de s'y attendre. Il faut noter cependant l'absence de support du réseau social. 


\section{CONCLUSION}

La présente étude a tenté de décrire les problèmes rencontrés par les sujets considérés comme à risque suicidaire, c'est-à-dire ceux qui ont déjà effectué une tentative de suicide ou sa planification. Elle voulait aussi cerner des sujets qui, même s'ils n'ont jamais été considérés par les auteurs parce qu'ils n'ont pas posé un geste dans ce sens, éprouvent néanmoins de nombreuses difficultés. La description de ce dernier groupe est particulièrement intéressante, compte tenu des problèmes rencontrés. En effet, ils présentent de nombreux problèmes farmiliaux, des éléments dépressifs et stressants, ainsi que les attitudes plutôt négatives face à la vie et à la mort. Ces patterns de comportement semblent assez similaires à ceux affichés par les sujets ayant déjả effectué une tentative de suicide.

L'intérêt de la présente étude réside dans le fait qu'elle aura tenté, sinon permis, de mieux spécifier le terme vague des «ruminations» utilisé par l'ensemble des auteurs en sortant des sentiers battus. Il est évident que, d'une part, le petit nombre de sujets interrogés et, d'autre part, l'absence d'un échantillonnage représentatif rend toute généralisation des résultats impossible. De futures recherches pourraient être effectuées avec des échantillons représentatifs afin d'arriver à gennéraliser les résultats. Une autre limite réside dans le fait que l'étude n'a mesurée que le support du réseau social et il aurait été intéressant de déterminer la constitution du réseau social comme tel et d'établir des comparaisons.

Les informations recueillies dans le cadre de la présente étude auront permis de mettre sur pied une intervention préventive au Cégep de Shawinigan qui revêt des caractéristiques différentes de ce qui se faisait auparavant. En effet, il ressort de la présente étude que les sessions d'information traditionnelles risquent de s'avérer inefficaces puisque la clientèle à risque élevé ne se sent pas concernée par le problème. Enfin, ces sessions ne sont pas plus efficaces pour les sujets qui ont déjà fait une tentative ou une planification parce qu'ils ont déjà été confrontés au problème et ont été obligés de trouver une solution. La journée d'information au Cégep de Shawinigan s'est donc orientée vers la présentation des caractéristiques que revêt toute personne à risque suicidaire ainsi que des services disponibles. L'animation allait dans le sens de sensibiliser les étudiants sur la façon dont se comporte une personne avant de passer à l'acte, de façon à ce qu'ils soient à même d'identifier ces personnes dans leur entourage immédiat ou même de se reconnaître. L'information sur les services disponibles leur permettaient de ne pas se sentir démunis et de savoir quoi faire. II y aurait aussi lieu de penser à des groupes de croissance dans le but de développer la capacité d'établir des relations chez les sujets à risque.

\section{NOTES}

1. L'élaboration et la validation des items et des échelles se sont étroitement inspirées des démarches originales, c'est-à-dire: (a) traduction, (b) contre-validation de la traduction, (c) révision des traductions, (d) prétest auprès d'un nombre restreint de personnes,

et (e) validation auprés d'échantillons et analyses des items et échelles.

2. Deux démarches ont été effectuées pour valider le critère de sélection retenu ( 30 et plus au test sur les idéations suicidaires): (a) une analyse du contenu du test a été effectuée 
par des cliniciens qui ont fixé à 30 le point de coupure pour discriminer les sujets ayan des difficultés au niveau des attitudes face à la vie. Ce jugement est confirmé par les résultats de l'étude puisque la moyenne du groupe A (tentative de suicide) à ce test es: effectivement de 30; (b) une analyse discriminante a été effectuée sur les données. Les indices statistiques se sont avérés significatifs $(p=, 0001)$ avec une valeur propre de 1,06, une corrélation canonique de ,72, un Lambda de Wills de ,47, et un Khi-carré de $234,9(\mathrm{~d}, f .=10)$. Ces résultats permettent de confirmer le classement puisque $89 \%$ des sujets considérés uà risque» présentent effectivement des «patrons» de comportements similaires à ceux des sujets des groupes A et B.

3. La moyenne harmonique a été utilisée dans toutes les analyses car elle permet de cor. riger la différence importante du nombre de sujets dans les groupes.

\begin{abstract}
The present article reports the results of a study of 550 Shawinigan Cégep students enrolled in their first year of college. The study attempts to establish the prevalence of suicidal behaviours and to describe predisposing factors identified by the authors such as family problems, lack of social support, depression, stressful events having a negative impact, and attitudes toward life and death. The information was gathered using a questionnaire combining several elements: (a) a questionnaire similar to the one used by Lamontagne (1986) and Tousignant, Bastien, Hamel, and Hanigan (1986) to identify family problems; (b) the Sarason, Levine, Basham, and Sarason (1983) Social Support Questionnaire, translated and adapted by De Man (1986), which evaluates social support; (c) the Aneshensel, Clark, and Fredrichs test (1983) which indicates the presence or absence of depression; (d) the Sarason, Johnson, and Siegel (1978) Life Experience Survey, measuring the quantity of stressful events experienced, and their impact on personal life; and (e) Beck's (1979) Scale of Suicide Ideation which measures attitudes toward life and death. Two questions were added for the present study: Did you ever attempt suicide? If yes, how many times?

Three hundred and fifty-five students answered the questionnaire, making the level of participation $71 \%(355 / 550)$. Participants were divided into four groups: those having made a suicidal attempt $(n=28)$ or planned suicide $(\mathrm{n}=16)$, those having a score of 30 or more on the test relating to suicidal ideation $(\mathrm{n}=18)$, and those regarded as "normal" $(\mathrm{n}=278)$. The cut-off point of 30 on the scale of suicidal ideation was determined by averaging the scores of those who made a suicide attempt and confirmed by a discriminant analysis. The students who had attempted suicide had major family problems, lacked social support, presented depressive tendencies, and had experienced stressful events having a negative impact on their lives. Furthermore, they manifested problems in their attitudes toward life and death. However, the study did not determine the contribution of each variable. The participants who had planned suicide, at first sight, did not seem to present more problems than those designated "normal," which seems astonishing. This was explained by the fact that the planned suicide was not recent or was a cry for help which found a response. Participants with a score of 30 or more on the test of suicidal ideation are considered at high risk because they present problems similar to those who have attempted suicide. Finally, as would be expected, no major problems were presented by the subjects classified as "normal."
\end{abstract}


The present study has attempted to describe the difficulties encountered by participants presenting problems, that is to say, those who have attempted or planned suicide; and to identify those who up to now, have not been considered at risk because they have never made an attempt, but who are experiencing difficulties and in certain cases, are veritable "time bombs waiting to go off." Despite the fact that the participants did not constitute a representative sample of the Quebec population, the results confirm those obtained in previous studies identifying predisposing factors in suicide. The information gathered in the course of the present study has enabled us to implement preventive intervention at the Shawinigan Cégep.

\section{REFERENCES}

Aneshensel, C.S., Clark, V.A., \& Fredrichs, R.S. (1983). Race, ethnicity, and depression: A confirmatory analysis. Journal of Personality and Social Psychology, 44, 385-398.

Beck, A.T., Kavacks, M., \& Wiessman, A. (1979). Assessment of suicide intention: The scale of suicide ideation. Journal of Consulting and Clinical Psychology, 47, 343-352.

Bonner, R.L., \& Rich, A.R. (1987). Toward a predictive model of suicidal ideation and behaviour: Some preliminary data in college students. Suicide and Life Threatening Behavior, 17, 50-63.

Bouchard, L. (1987). Incidences des comportements suicidaires chez les étudiants de l'Université de Montréal. Mémoire de maîtrise inédit, Université de Montréal, Montréal, PQ.

Carlson, G.H., \& Cantwell, D.P. (1982). Suicidal behavior and depression in children and adolescents. Journal of the Academy of Child Psychiatry, 2l(4), 361-368.

Chabrol, H. (1984). Les comportements suicidaires de l'adolescent. Paris: Presses Universitaires de France.

Charron, W.F. (1981). Le suicide au Québec: Analyses statistiques. Québec: Gouvernement du Québec.

Corbeil, S.C. (1984). Suicide et adolescence, Dans P. Morissette (Ed), Le suicide (p. 46). Québec: Pierre Morissette.

Davidson, F., \& Choquet, M. (1972). Contribution à l'étude du suicide des adolescents: Etude médico-sociale de 139 tentatives de suicide, Hygiène Mentale, 61(1), 1-31.

Davidson, F., \& Philippe, A. (1986). Suicide et tentative de suicide aujourd 'hui, étude épidémiologique. Nancy: Les Editions INSERS Dorin.

Davis, P.A. (1983). Suicidal adolescents. Springfield, IL: Charles C. Thomas.

De Man, A.F. (1986). Aide naturelle d̀ la personne suicidaire en Estrie. Rapport non publié. Sherbrooke, PQ: Département de Santé communautaire du Centre hospitalier de l'Université de Sherbrooke.

Faber, M.L. (1968). Theory of suicide. New York: Funk and Wagnalls.

Farberow, N.L. (1985). Youth suicide: An international problem. Report of the National Conference on Youth Suicide. Washington, DC.

Friedman, J.M.H., Gregory, A.M., Boeck, M., \& Difiore, J. (1987). Prevalence of specific suicidal behaviour in a high school sample. American Journal of Psychiatry, 144 (9), 1203-1206.

Gaulthier, M., Fournier, E., \& Gorceix, A. (1961). A propos de 47 cas de tentatives de suicide chez les adolescents. Revue Hygiène Mentale, 50(6), 49-57.

Grégoire, J.C., \& Phaneuf-Perron, L. (1980). Le suicide, considération d'ordre théorique et apençu des types et méthodes d'intervention. Montréal: Editeur Officiel du Québec.

Haim, A. (1969). Le suicide chez les adolescents. Paris: Payot.

Harlow, L. L., Newcomb, M.D., \& Bentler, P.M. (1986), Depression, self-derogation, substance use, and suicide ideation: Lack of purpose in life as a mediational factor. Journal of Clinical Psychology, 42(1), 5-21. 

Hertzman, M. (1985). Alcohol, drug and adolescent suicide. Report of the National Con.
ference of Youth Suicide, Washington, DC. Jenicek, M. (1975). Epidémiologie, principes, techniques, application. Note de cours.
Montréal: Université de Montréal.

Joan, P. (1986). Preventing teenage suicide. New York: Human Sciences Press.

King, A.J.C. (1986). The adolescent experience. Toronto: The Ontario Secondary School
Teacher's Federation.

Ladame, F. (1981). Les tentatives de suicide des adolescents. Paris: Masson.

Lamontagne, Y. (1986). Suicide et dépression chez les étudiants de C.E.G.E.P. L'Union mé. dicale du Canada, 522.

Morissettc, P. (1984). Le suicide: Démystification, intervention, prévention. Québec: Edi. tions Garotex.

Noles, S.W., Cash, T.F., \& Winstead, B. (1985). Body image, physical attractiveness and depression. Journal of Consulting and Clinical Psychology, 53, 88-94.

Pokorny, A.D. (1960). Characteristics of 44 patients who subsquently committed suicide, Archives of General Psychiatry, 2, 314-323.

Quidu, M. (1970). Le suicide, étude clinique, perspectives préventives. Paris: Editions Sociales Françaises.

Radloff, L.S. (1977). The C.E.S.D. Scale, a self-report depression scale for research in the general population. Applied Psychological Measurement, I(3), 385-401.

Santé et Bien-être social Canada. (1987). Le suicide au Canada. Ottawa: Editeur Officiel du Canada.

Sarason, G., Johnson, I.H., \& Siegel, M. (1978). Assessing the impact of life changes: D velopment of life experience survey. Journal of Consulting and Clinical Psychology, 46, 936-946.

Sarason, G., Levine, H.M., Basham, R.B., \& Sarason, B.R. (1983). Assessing social support: The Social Support Questionnaire. Journal of Personality and Social Psychology, 44, 127-139.

Teicher, J.D., \& Jacobs, J. (1966). The physician and the adolescent suicide attempter. Journal of School Health, 36(9), 406-415.

Tishler, C.L., Carl, L., McKenny, P.L., \& Morgan, K.C. (1981). Adolescent suicide attempt: Some significant factors. Suicide and Life-Threatening Behavior, II (2), 89-92.

Tousignant, M., Bastien, M.F., Hamel, S., \& Hanigan, D. (1986). Comportements et idéations suicidaires chez les cégépiens de Montréal: La part de la famille. Apprentissage et socialisation, 9I, 17-25.

White, R.H. (1963). The study of lives. New York: Prentice Hall.

Withers, L.E., \& Kaplan, D.W. (1987). Adolescents who attempt suicide: A retrospective clinical chart review of hospitalized patients. Profession and Psychology Research and Practice, 18(4), 391-393. 


\title{
INVITATION A SOUMETTRE UN ARTICLE
}

\author{
Numéro spécial, automne 1992 \\ Revue Canadienne de Santé Mentale Communautaire
}

le thème porte sur

\begin{abstract}
Travail, vie privée, et santé mentale: le rôle nouveau du communautaire
\end{abstract}

Les rapports entre l'organisation du travail et la santé mentale des travailleurs et travailleuses font enfin l'objet, et ceci depuis quelques années à peine, d'un travail de recherche significatif. Les directions d'entreprise, les syndicats, et les gouvernements abordent également, non sans hésitation, cette question: comment favoriser de meilleures conditions de vie au travail et une plus grande santé mentale chez les travailleurs et travailleuses? D'un autre côté, les organismes de santé reconnaissent de plus en plus l'importance d'intégrer la vie au travail dans les stratégies de promotion de la santé mentale dans la communauté et de prévention de la maladie.

L'organisation du travail et son lien avec la communauté sont reconnus de plus en plus comme des facteurs importants de la qualité de vie des gens, en particulier dans le développement de leur capacité de prise en charge. Les recherches tendent à indiquer que le bien-être de l'individu est lié à la possibilité sociale de pouvoir satisfaire un besoin comme celui d'occuper un emploi suffisamment rémunéré pour assurer un mode de vie décent. Plus encore, les structures décisionnelles favorisant la participation démocratique dans son milieu de travail et dans d'autres milieux institutionnels sont une nécessité vitale pour assurer le plein développement humain. Ceci semble se traduire au niveau de la santé mentale, de l'espérance de vie, et de la qualité du développement psychosocial.

Le premier objectif de ce numéro est de rendre compte de l'état actuel des recherches dans ce secteur nouveau, et ceci pour l'ensemble du Canada. Un second objectif est de dégager les principaux éléments d'une problématique de soutien social et communautaire à la santé mentale, reliant milieux de travail et vie hors travail, y compris la situation des sans travail. Un troisième objectif est de faire connaître les programmes d'action et les stratégies les plus prometteuses dans l'établissement des mesures positives de soutien social à la santé mentale au travail.

Plusieurs thèmes peuvent être abordés pour couvrir cet ample territoire. En voici quelques exemples:

- effets de l'organisation du travail sur la santé mentale des travailleurs et travailleuses;

- les nouvelles approches communautaires et le travail : coopératives de développement économique, réseaux de support au travail et à l'extérieur, économie informelle; 
- les programmes d'aide aux employés;

- l'action syndicale dans le dossier de la santé mentale au travail;

- les stratégies de participation et de réorganisation du travail et leurs rapports à la santé mentale;

- la problématique des liens entre travail, santé mentale, réseaux sociaux au travail et dans la vie privée;

- alcoolisme ou toxicomanie, travail, et santé mentale;

- chômage, travail, et santé mentale;

- la psychopathologie du travail;

- les rôles multiples des femmes au travail et la santé mentale;

- obstacles à l'égalité au travail pour les femmes, les minorités ethniques, les minorités sexuelles;

- obstacles à l'équité salariale;

- santé, sécurité, et santé mentale;

- stress, burn out, et santé mentale;

- qualité de vie, travail, et santé mentale.

Les articles doivent répondre aux critères habituels pour l'acceptation de tout manuscrit soumis à la revue. Dans le but de favoriser le dialogue avec les lecteurs anglophones, les auteurs de manuscrits rédigés en français peuvent, s'ils le désirent, produire un sommaire substantiel d'environ 1000 mots.

Echéancier pour la soumission d'un projet d'article: le ler octobre 1991. Echéancier pour la soumission d'un manuscrit : le 11 décembre 1991. Les articles rédigés en français seront envoyés a :

Jacques Rhéaume

Département des communications

Université du Québec à Montréal

C.P. 8888 , Succ. A.

Montréal, PQ H3C 3P8 